\title{
DESARROLLAR CONCEPTOS DE FÍSICA A TRAVÉS DEL TRABAJO EXPERIMENTAL: EVALUACIÓN DE AUXILIARES DIDÁCTICOS
}

\author{
BERNARDINO LOPES, J. \\ Departamento de Física. UTAD. Universidad de Trás-os-Montes e Alto Douro \\ Quinta de Prados, 5000 Vila Real. Portugal \\ blopes@utad.pt
}

\begin{abstract}
Resumen. El aprendizaje conceptual a través del trabajo experimental es un problema abierto tanto para la investigación didáctica como para la práctica docente. Estudios sobre la naturaleza del aprendizaje de física indican que no sólo es posible, sino también deseable promover el desarrollo conceptual a través del trabajo experimental, estando éste integrado en el currículo. Dentro de este trabajo se evalúa la relevancia de auxiliares didácticos para promover el desarrollo conceptual a través del trabajo experimental y su utilización por profesores. El contexto utilizado es la administración en la clase de actividades experimentales en los dominios de la mecánica y de la acústica. Dichos auxiliares didácticos permiten aclarar las entidades y estructuras conceptuales en juego en cada actividad experimental. Los profesores han reconocido la extrema importancia de tal recurso didáctico y los resultados del aprendizaje evidencian que dan lugar al desarrollo de competencias no elementales en la utilización de conceptos implicados en los trabajos experimentales.
\end{abstract}

Palabras clave. Mecánica, acústica, trabajos prácticos, aprendizaje de conceptos, desarrollo curricular.

Summary. Conceptual learning through experimental work is an issue open to both didactic research and practical teaching. Studies on the nature of Physics learning show that it is not only possible, but also desirable to foster conceptual development through experimental work, the latter being inserted in the curriculum. Within this paper we assess the relevance of didactic assisting elements to foster conceptual development through experimental work and its use by teachers. The context used is the implementation in the classroom of experimental activities in the fields of Mechanics and Acoustics. These didactic assistants allow to clarify the entities and conceptual structures involved in every experimental activity. Teachers acknowledge the utmost importance of such a didactic resource, and the results in learning show that they result in non-elementary competence when using the concepts involved in the experimental work.

Keywords. Mechanics, acoustics, practical work, learning of concepts, curriculum.

\section{CONTEXTO DEL ESTUDIO}

La investigación didáctica sobre el trabajo experimental (TE) en física demostró que existen serias dificultades en el desarrollo conceptual a través del TE (Hodson, 1994). Por otro lado, concretamente en Portugal, el TE es alentado por algunos programas gubernamentales y su utilidad es, en general, fácilmente reconocida. Existe otro tipo de estudios que identificaron la unión estrecha entre la cognición de un sujeto, su actividad sobre situaciones físicas y la manipulación sobre sistemas simbólicos como esenciales para la construcción conceptual (Vergnaud, 1987; Lemeignan et al., 1993). Por consiguiente, el aprendizaje conceptual a través del TE es un asunto relevante desde el punto de vista de la investigación y de la práctica educativa. Este marco permite cuestionar el aprendizaje conceptual en los moldes tradicionales. Este trabajo se encuadra, también, en el esfuerzo de transformar la investigación didáctica en TE útil en y para la clase como ejemplo de varios autores (García et al., 1995; Sánchez et al., 1997; Pro Bueno, 1999).

\section{Marco teórico y problema de la investigación}

El marco teórico en el que nos basamos se apoya en tres aspectos: $a$ ) la formación de conceptos de física; $b$ ) la 
naturaleza evolutiva de su aprendizaje; y c) el papel del profesor en el proceso de construcción del conocimiento.

En relación con la formación de los conceptos de física consideramos las aportaciones de Vergnaud $(1987,1991)$ y Lemeignan y Weil-Barais (1993). En efecto, una de las principales características evidenciadas es que el aprendizaje conceptual se apoya en la acción de los sujetos sobre situaciones físicas, de forma articulada y en relación con la actividad cognitiva, en concreto con la utilización de lenguaje para comunicar y operar sobre entidades conceptuales y la utilización y construcción de relaciones, propiedades. De acuerdo con estos estudios tiene poco sentido continuar separando conocimiento conceptual de procedimental. Así, el TE puede ser visto en el contexto del aprendizaje conceptual de forma más clara y consecuente. Según aquellos autores, el concepto físico es un tripleto de componentes articulados entre sí: conjunto de situaciones físicas y cuestiones (que se reconoce, manipula, controla, opera y que da sentido a los conceptos físicos), conjunto de lenguajes simbólicos asociados (que permite traducir, operar y comunicar sobre los conceptos en referencia a una situación física y cuya semántica y pragmática se domina) y conjunto de propiedades, relaciones y reglas de acción inherentes a los conceptos. De esta forma, los conceptos son construidos y utilizados en referencia a un conjunto de situaciones físicas que progresivamente deberá ser extendido.

En este marco, los conceptos no son independientes, están asociados entre sí constituyendo una entidad designada como campo conceptual y es éste el que se desarrolla. O sea, sólo tenemos verdadero desarrollo conceptual si se tienen en cuenta las relaciones intrincadas entre los varios conceptos que son relevantes para tratar determinada clase de situaciones físicas. Este desarrollo no puede hacerse sólo en la vertiente de los lenguajes simbólicos utilizados en la comunicación y en la operación sobre los propios conceptos (incluso enriquecidos y articulados entre sí), tiene que asentar también y concomitantemente, en la acción de los sujetos sobre las situaciones físicas (en concreto las experimentales) y en la extensión y estructuración de propiedades, relaciones y reglas de acción relativas a los conceptos que se utilizan y reconocen. De esta forma, la actividad experimental, en sentido amplio, tiene nuevos contornos y su implantación en la clase sitúa nuevas potencialidades y desafíos.

Así, el TE es una ocasión privilegiada para construir y desarrollar conceptos, pues al mismo tiempo que moviliza conceptos será necesario reformular algunos, enriquecer y eventualmente aprender otros. Para situaciones más complejas más allá de los conceptos es necesario construir o utilizar un modelo físico que recurra a una conceptualización de la situación física, la cual tiene que ser trabajada, -necesita una movilización de conceptos-, hasta que se elabore un modelo y sea calculable (Martinand, 1995; Larcher, 1996, Lopes et al. 1999). Es necesario considerar ciertas especificaciones del TE de física (tales como el tratamiento de los datos, la construcción de una relación funcional, etc.) que, al contrario de lo que pueda parecer, son además de naturaleza conceptual (Journeaux, 1996).

La naturaleza evolutiva del aprendizaje de los conceptos de física ha sido puesta en evidencia por diversos tipos de investigación (Silva, 1999; Lopes et al., 1999). Concretamente, no se puede esperar que de un momento a otro un alumno pueda utilizar un campo conceptual como al profesor le gustaría que hiciese sólo porque trabajó con él ese campo conceptual un cierto tiempo. Así, los esfuerzos considerables que la corriente de investigación muchas veces designada como concepciones alternativas hizo y ha hecho para ultrapasar las recurrentes dificultades de los alumnos en el aprendizaje de física (Viennot, 1996) están envueltos, aparentemente, en una contradicción teórica: no puede haber cambio conceptual si el aprendizaje es de naturaleza evolutiva. La investigación didáctica está buscando nuevos paradigmas que, además de explicar, permitan intervenir en el aprendizaje de física (Weil-Barais, 1995), teniendo en cuenta la naturaleza evolutiva del aprendizaje de física. Este trabajo se inserta en ese esfuerzo.

El profesor tiene un papel determinante en el enriquecimiento de los aprendizajes conceptuales de los alumnos (Vygotsky, 1934). En el seguimiento de las nuevas perspectivas sobre la comprensión del aprendizaje de física surge como fundamental, incluso integrado en un contexto más amplio, el papel determinante del profesor para enriquecer los aprendizajes conceptuales de los alumnos (Franceschelli et al., 1998). El profesor surge como un mediador de aprendizajes, el cual, además de eso, ha asociado un estatuto de «representante legal del conocimiento establecido». La investigación conducida por Dumas-Carré y otros (1998), a pesar de ser en resolución de problemas de papel y lápiz, es explicativa de esta problemática.

Nótese que los tres aspectos esenciales del marco teórico presentado convergen para la revalorización del papel mediador del profesor, adquiriendo éste una importancia acrecentada en la enseñanza y en el aprendizaje de física (Dumas Carré et al., 1998; Vygotsky, 1934).

En síntesis, los conceptos no pueden ser presentados y trabajados sólo en su dimensión simbólica (y en ésta casi sólo en el dominio de las matemáticas), sino en interrelación con la actividad del sujeto, mediada por el profesor, en un referente empírico, el cual engloba la actividad experimental (Lemeignan et al., 1993; Vergnaud, 1987, 1991; Lopes, 1999). Sólo de esta forma es posible que se construya un campo conceptual y condiciones para que se modelen situaciones físicas. La actividad experimental, en la medida en que permite una acción del sujeto sobre un referente empírico, posibilita que su campo conceptual se estructure y enriquezca, en particular en términos de modelos de utilización de los conceptos.

Por consiguiente, el problema de investigación es: ¿de qué modo se pueden desarrollar competencias variadas relativas a la utilización de conceptos de física utilizan- 
do el trabajo experimental clásico potenciado por auxiliares didácticos apropiados?

\section{Caracterización del modelo de construcción de auxi- liar didáctico}

En el contexto referido anteriormente, el estudio de la utilización y la utilidad, en clase, de auxiliares didácticos en la exploración de un TE y en la promoción del desarrollo conceptual de los alumnos asume una importancia fundamental. Así, se concibió, como hipótesis de trabajo, para cada actividad experimental un «auxiliar didáctico del desarrollo conceptual a través del trabajo experimental» (ADDCTE), a partir de un modelo general (Lopes et al., 2000). El modelo adoptado para elaborar un ADDCTE asociado a un protocolo experimental (Cuadro I) se asienta en el marco teórico anteriormente presentado y tiene cuatro características fundamentales:

A. La experiencia propuesta en el protocolo está asociada a una situación física, más o menos académica, que es necesario explicitar para que el campo de acción sea consciente y deliberado (punto 1 del modelo de estructura del ADDCTE).
$B$. La acción y el pensamiento sobre la situación física, objeto de experimentación, moviliza un campo conceptual con una determinada estructura y, en concreto, con determinados conceptos. Esta necesidad de movilización es una oportunidad para la (re)construcción conceptual de los alumnos a partir de su propio campo conceptual, siempre que sea adecuadamente guiada por el profesor. Por esto es necesario inventariar los conceptos, identificar las relaciones entre ellos, identificar los aspectos operacionales necesarios en la manipulación de los conceptos (punto 2 del modelo de estructura del ADDCTE), explicitar el modelo teórico necesario, tanto el modelo del sistema físico como del abordaje experimental (punto 3) y proponer una orientación para la evaluación del profesor antes, durante y después del TE propiamente dicho (punto 4 ).

C. El protocolo experimental tiene que ser flexible a las acciones de los alumnos, al transcurso de los trabajos e incluso a la exploración de otros aspectos interesantes que pueden hacer puente con otras situaciones físicas. De esta forma, la indicación de variantes al protocolo inicial permite una flexibilidad mental útil para la evaluación del profesor y para la extensión de los aprendizajes (punto 5).

1) Situación física y particularidades

(Describir la situación física y particularidades de su abordaje)

2) Conceptos en juego

(Lista exhaustiva de los conceptos, conceptos centrales, relaciones entre conceptos y aspectos operacionales de su uso)

3) Modelo implícito

(Indicación de los aspectos esenciales de modelo(s) físico(s) implícito(s) de la situación física tratada en el protocolo y de los modelos teóricos necesarios al abordaje experimental)

4) Desarrollo conceptual durante la realización del protocolo

\begin{tabular}{|l|l|l|}
\hline Estructura del protocolo & Sugerencias de exploración & Desarrollo conceptual \\
\hline Situación física & $\begin{array}{l}\text { - Referir las sugerencias dadas en } \\
\text { el protocolo } \\
\text { - Incluir otras sugerencias } \\
\text { - Referir notas relativas a la calidad } \\
\text { del trabajo a efectuar por los alumnos }\end{array}$ & $\begin{array}{c}\text { - Explicitar los procesos } \\
\text { conceptuales en causa } \\
\text { - Explicitar los principales pasos } \\
\text { a seguir obligatoriamente (procesos } \\
\text { de representación, operaciones } \\
\text { o manipulaciones, etc.) } \\
\text { - Explicitar el modelo en causa }\end{array}$ \\
Problema & Ídem & Ídem \\
\hline $\begin{array}{l}\text { (Principales } \\
\text { pasos del protocolo) }\end{array}$ & Ídem & Ídem \\
\hline
\end{tabular}

5) Variantes del protocolo (Señala algunas variantes)

6) Comunicación

(Sugerencias para estructurar la comunicación, formas de efectuar la comunicación, enfoque de los principales aspectos que se retuvieron durante el aprendizaje) 
$D$. La comunicación de lo que se ha hecho y de lo que se ha aprendido es otra dimensión importante del aprendizaje, pues tiende a explicitar aspectos que durante la acción estaban implícitos y, por consiguiente, a concienciar el aprendizaje (punto 6).

Los ADDCTE son herramientas didácticas elaboradas a partir de los protocolos experimentales tradicionales que se pueden prever para una unidad de enseñanza. Son herramientas para el profesor y no para los alumnos. El proceso de elaboración de los ADDCTE puede provocar modificaciones en la estructura de los protocolos previamente elaborados y desencadenar algunos ajustes en la planificación. Así, los ADDCTE tienen esencialmente dos funciones: ayudar a reflexionar y a mejorar la planificación de la enseñanza experimental del profesor y servir de referencia para la gestión de las actividades experimentales en la clase incluyendo su discusión.

Los ADDCTE son herramientas didácticas que permiten explicitar las especificaciones didácticas y científicas de los aspectos conceptuales y empíricos involucrados en la actividad experimental. En particular permiten explicitar las entidades y las estructuras conceptuales involucradas en una actividad experimental en el ámbito de la física. Pueden ayudar los profesores a tomar conciencia de esos aspectos, facilitando una articulación entre aspectos teóricos y experimentales tradicionalmente separados y articular las actividades experimentales y las restantes actividades de enseñanza con el objetivo de un aprendizaje más eficaz. No obstante, los ADDCTE no son guiones, en sentido literal, ni herramientas listas para ser utilizadas y, por tanto, no son prescriptibles.

\section{Objetivo del trabajo}

El principal objetivo de este trabajo es evaluar la importancia, utilidad y condiciones de utilización de los ADDCTE como herramienta didáctica para promover el desarrollo conceptual de conceptos físicos, de un determinado dominio, a través del trabajo experimental.

\section{DESCRIPCIÓN DEL ESTUDIO}

El estudio consistió en la producción de un ADDCTE para cada protocolo experimental, en la implantación de trabajo experimental en contenidos de física y niveles de enseñanza diferentes (proporcionando un protocolo experimental a los alumnos y utilizando, el profesor, el respectivo ADDCTE) y en la evaluación de la importancia, utilidad y condiciones de utilización de los ADDCTE.

El estudio tuvo tres fases: $a$ ) antes de la implantación en la clase; $b$ ) durante la implantación en la clase; y c) análisis y discusión de los resultados del aprendizaje de los alumnos.

Participaron en el estudio cuatro profesores y cuatro grupos, uno de cada profesor. Dos profesores, A y B, impartían clases en la enseñanza secundaria. Los otros dos, C y D, en la enseñanza básica. La caracterización sucinta de los profesores, de los temas que explicaron y de los grupos que participaron en el estudio se encuentra en el cuadro II. Participó también un investigador en las tres fases del estudio. Además, el investigador no estuvo presencialmente en las clases.

\section{Antes de la implantación en la clase}

Los protocolos experimentales empezaron a ser elaborados, anteriormente a los ADDCTE, por los profesores involucrados y fueron objeto de trabajo conjunto (profesores y investigador), del cual resultó una explicitación de las características de los protocolos y su perfeccionamiento. Fueron utilizadas cuatro sesiones de trabajo de tres horas. Las experiencias que se componían de los protocolos fueron seleccionadas, por los profesores, de manera que contemplasen aspectos claves de la unidad curricular en la que estaban insertados y que estuviesen integrados de forma plena en los currículos (Protocolo experimental, anexo A).

Los ADDCTE fueron concebidos, a partir de los protocolos experimentales, durante un largo proceso de debate y de mejoras progresivas, que se basaban en el modelo de estructura de los ADDCTE (Cuadro I y anexo B como

\begin{tabular}{|l|l|c|c|}
\hline & Experiencia profesional & Tema y nivel de enseñanza & Núm. de alumnos \\
\hline Profesor A & 25 años de servicio + varios cargos & Mecánica $\left(10^{\circ}\right.$ curso $-15-16$ años $)$ & 26 alumnos \\
\hline Profesor B & 15 años de servicio + varios cargos & Mecánica $\left(10^{\circ}\right.$ curso $-15-16$ años $)$ & 20 alumnos \\
\hline Profesor C & 10 años de servicio + formación posgraduada & Acústica $\left(8^{\circ}\right.$ curso $-13-14$ años $)$ & 26 alumnos \\
\hline Profesor D & 9 años de servicio + formación posgraduada & Acústica $\left(8^{\circ}\right.$ curso $-13-14$ años $)$ & 20 alumnos \\
\hline
\end{tabular}


Cuadro III

Trabajos experimentales realizados

\begin{tabular}{|c|l|l|}
\hline & Mecánica $\left(\mathbf{1 0}^{\mathbf{}}\right.$ curso $)$ & Acústica $\left(\mathbf{8}^{\mathbf{}}\right.$ curso $)$ \\
\hline TE 1 & Medición de magnitudes físicas & Naturaleza y características del sonido \\
\hline TE 2 & Determinación del valor del trabajo realizado por una fuerza variable & Representación física del sonido \\
\hline TE 3 & Ley del trabajo-energía & Límites de audición \\
\hline
\end{tabular}

ejemplo de ADDCTE). Se utilizaron seis sesiones de tres horas. Fueron construidos de modo que influenciasen la gestión del TE y la discusión de los resultados y de las conclusiones. De esta forma los conceptos subyacentes podrían desarrollarse, en los alumnos, en las vertientes de los lenguajes necesarios para su utilización, en las propiedades y relaciones con otros conceptos, en las diferentes particularidades que pueden tener en distintas situaciones de una clase de contextos y en los modelos físicos intermediarios entre los resultados experimentales y las previsiones teóricas.

\section{Descripción de la implantación del trabajo experi- mental en la clase}

En mecánica, la implantación del trabajo experimental en la clase fue realizada por dos profesores (A y B) y sus respectivas clases. Transcurrió durante el año lectivo de 1998-99 en una escuela de una ciudad del norte de Portugal. En acústica, la implantación del trabajo experimental en la clase fue realizada por dos profesores (C y D) y sus respectivas clases. Transcurrió durante el año lectivo de 1999-2000 en dos escuelas de dos ciudades en el norte de Portugal.
El TE fue implementado en cada grupo de estudio por el respectivo profesor sin observación externa. Cada profesor se comprometió a hacer un diario que reflejase, de alguna manera, cómo transcurrieron las clases y cuáles fueron las impresiones con las que se quedaron, y la forma cómo los ADDCTE sirvieron para mediar el trabajo de los alumnos antes, durante y después de haber efectuado el trabajo propuesto en los procedimientos del protocolo experimental. Los trabajos experimentales realizados están indicados en el cuadro III.

La distribución de las clases dedicadas a los diferentes trabajos experimentales está representada en el cuadro IV.

La implantación de las actividades experimentales, en la clase, fue realizada de acuerdo con los criterios comparativos representados en el cuadro $\mathrm{V}$. En el caso de los profesores A y B, los TE se realizaron una vez que todos los aspectos teóricos sobre los asuntos tratados en el TE se habían abordado y consolidado con ejercicios. En los casos de los profesores C y D, las clases de trabajo experimental no se demarcaron tanto del enfoque teórico. En particular el TE num. 1 precedió un enfoque teórico sistematizado.

Cuadro IV

Distribución del número de clases por los diferentes trabajos experimentales.

\begin{tabular}{|c|c|c|c|c|}
\hline & Profesor A & Profesor B & Profesor C & Profesor D \\
\hline Total de clases & 32 (Mecánica) & 30 (Mecánica) & 21 (Acústica) & 10 (Acústica) \\
\hline Total de clases con TE & 6 & 4 & 8 & 5 \\
\hline TE núm. 1 & 3 & 2 & 3 & 1 \\
\hline TE núm. 2 & 1 & 1 & 2 & 2 \\
\hline TE núm. 3 & 2 & 1 & 3 & 2 \\
\hline Total de clases con otras actividades & 26 & 26 & 13 & 5 \\
\hline
\end{tabular}

Nota: Las discusiones de los resultados u observaciones realizadas por los profesores C y D transcurrieron durante la implantación del TE de acuerdo con los ADDCTE; los profesores A y B no efectuaron discusiones explícitas. 
Cuadro V

Características de la implantación de los TE y de la utilización de los ADDCTE por los diferentes profesores.

\begin{tabular}{|c|c|c|c|c|}
\hline $\begin{array}{l}\text { Criterios } \\
\text { comparativos }\end{array}$ & $\begin{array}{c}\text { Profesor A } \\
(26 \text { alumnos })\end{array}$ & $\begin{array}{l}\text { Profesor B } \\
(20 \text { alumnos })\end{array}$ & $\begin{array}{c}\text { Profesor C } \\
(26 \text { alumnos) }\end{array}$ & $\begin{array}{l}\text { Profesor D } \\
(20 \text { alumnos })\end{array}$ \\
\hline $\begin{array}{l}\text { Forma } \\
\text { de presentar } \\
\text { el protocolo } \\
\text { experimental }\end{array}$ & $\begin{array}{l}\text { Leído por el profesor } \\
\text { en voz alta }\end{array}$ & $\begin{array}{l}\text { Proporcionado a los } \\
\text { alumnos en la víspera de } \\
\text { serutilizado y explicación } \\
\text { de lo que se pretendía }\end{array}$ & $\begin{array}{l}\text { Cada parte del protocolo } \\
\text { sólo se presentaba } \\
\text { después de haber } \\
\text { concluido la anterior }\end{array}$ & $\begin{array}{l}\text { Cada parte del protocolo } \\
\text { sólo se presentaba } \\
\text { después de haber } \\
\text { concluido la anterior }\end{array}$ \\
\hline $\begin{array}{l}\text { Ejecución } \\
\text { del trabajo } \\
\text { propuesto } \\
\text { en el protocolo }\end{array}$ & $\begin{array}{l}\text { Los alumnos } \\
\text { siguieron el } \\
\text { protocolo y tomaron } \\
\text { notas. La memoria se } \\
\text { efectuó fuera de la } \\
\text { clase }\end{array}$ & $\begin{array}{l}\text { Los alumnos siguieron } \\
\text { el protocolo y tomaron } \\
\text { notas. La memoria se } \\
\text { efectuó fuera de la clase }\end{array}$ & $\begin{array}{l}\text { Los alumnos ejecutaban } \\
\text { las tareas y efectuaban } \\
\text { registros. En cada } \\
\text { momento el profesor } \\
\text { hacía una síntesis }\end{array}$ & $\begin{array}{l}\text { Los alumnos ejecutaban } \\
\text { las tareas. En cada } \\
\text { momento el profesor } \\
\text { hacía una síntesis }\end{array}$ \\
\hline $\begin{array}{l}\text { Organización } \\
\text { del trabajo }\end{array}$ & $\begin{array}{l}\text { Trabajo de grupo } \\
\text { (4 grupos) }\end{array}$ & $\begin{array}{l}\text { Trabajo de grupo } \\
\text { (3 grupos) }\end{array}$ & $\begin{array}{l}\text { Trabajo de grupo } \\
\text { (4 grupos) }\end{array}$ & $\begin{array}{l}\text { Trabajo de grupo } \\
\text { (4 grupos) }\end{array}$ \\
\hline $\begin{array}{l}\text { Papel } \\
\text { del profesor }\end{array}$ & $\begin{array}{l}\text { Estar atento y ayudar } \\
\text { cuando sea necesario }\end{array}$ & $\begin{array}{l}\text { Estar atento y ayudar } \\
\text { cuando sea necesario }\end{array}$ & $\begin{array}{l}\text { Orientar los trabajos, } \\
\text { ayudar cuando sea } \\
\text { necesario, orientar la } \\
\text { elaboración de las } \\
\text { síntesis }\end{array}$ & $\begin{array}{l}\text { Orientar los trabajos, } \\
\text { ayudar cuando sea } \\
\text { necesario, orientar la } \\
\text { elaboración de las síntesis }\end{array}$ \\
\hline $\begin{array}{l}\text { Condicionantes } \\
\text { señalados }\end{array}$ & Falta de tiempo & Falta de tiempo & $\begin{array}{l}\text { Falta de laboratorio y } \\
\text { escasez de material } \\
\text { frente al número de } \\
\text { alumnos }\end{array}$ & Alumnos desinteresados \\
\hline $\begin{array}{l}\text { Forma de utilizar } \\
\text { los ADDCTE }\end{array}$ & $\begin{array}{l}\text { Sirvió de base para } \\
\text { la exploración de la } \\
\text { experiencia. } \\
\text { Guió las } \\
\text { observaciones } \\
\text { hechas con relación } \\
\text { al trabajo que estaba } \\
\text { siendo desarrollado } \\
\text { por los alumnos, } \\
\text { durante las clases } \\
\text { experimentales }\end{array}$ & $\begin{array}{l}\text { Sirvió de base para las } \\
\text { ayudas durante la } \\
\text { realización de la } \\
\text { experiencia. } \\
\text { Guió la verificación de } \\
\text { si los alumnos estaban } \\
\text { adquiriendo los } \\
\text { conceptos } \\
\text { confrontándolos con sus } \\
\text { propios modelos }\end{array}$ & $\begin{array}{l}\text { Permitió flexibilidad en } \\
\text { el trabajo con los } \\
\text { diferentes grupos y } \\
\text { articular los asuntos } \\
\text { tratados en todas las } \\
\text { clases } \\
\text { Ayudó a hacer la } \\
\text { articulación con otros } \\
\text { aspectos de la enseñanza }\end{array}$ & $\begin{array}{l}\text { Permitió articular los } \\
\text { asuntos tratados en todas } \\
\text { las clases } \\
\text { Ayudó la gestión de los } \\
\text { períodos entre tareas, al } \\
\text { final de las cuales el } \\
\text { profesor debería } \\
\text { intervenir de manera que } \\
\text { promoviese debate de los } \\
\text { conceptos en fase de } \\
\text { estudio }\end{array}$ \\
\hline
\end{tabular}

\section{Recogida y tratamiento de la información}

La información recogida fue la siguiente:

a) Relatos escritos realizados por los profesores sobre el modo cómo transcurrieron las clases a lo largo de la implantación del trabajo experimental y cómo utilizaron los ADDCTE, de los cuales resultó el cuadro V.

b) Opiniones escritas de los profesores sobre la importancia de los ADDCTE para la forma cómo se gestionaron las clases de TE y para el aprendizaje de los alumnos, de las cuales resultó el cuadro X.

c) Copias de las memorias efectuadas por los alumnos para cada TE. Cada grupo de alumnos elaboró una memoria. En $8^{\circ}$ curso las memorias se efectuaron integralmente en las clases. En $10^{\circ}$ curso las memorias se iniciaron en las clases y se concluyeron en casa. d) Análisis, a posteriori, de los aspectos conceptuales implícitos y explícitos de los protocolos experimentales y los ADDCTE relativos al TE núm. 1 de mecánica y al TE núm. 1 de acústica.

e) Test de evaluación de conceptos aplicado a los alumnos de $10^{\circ}$ curso. Se efectuó un pretest y un postest con los mismos aspectos conceptuales incluidos aunque con cuestiones efectivamente diferentes.

f) Opiniones escritas de los alumnos ( $8^{\circ}$ y $10^{\circ}$ cursos $)$ sobre la importancia de los trabajos experimentales para relacionar los conceptos explicados en las clases teóricas y teórico-prácticas, de las cuales resultó el cuadro IX.

De los puntos $a, b$ y $f$ se hizo una síntesis descriptiva expresada en los cuadros ya referidos. A partir del punto $e$ se hizo un análisis de las competencias de utilización de los conceptos requeridas a los alumnos de $10^{\circ}$ (columna de la izquierda del cuadro VII). 
A partir del análisis referido en $d$ se concretaron los aspectos conceptuales de mecánica o acústica a desarrollar que se encuentran explícitos en la columna de la izquierda de los cuadros VI y VIII respectivamente. Estos aspectos deberían encontrarse en las memorias del TE núm. 1 de los dos niveles de enseñanza. A pesar de que los TE núm. 2 y 3 abordan asuntos diferentes (Cuadro III), sería de esperar que los aspectos conceptuales referidos estuviesen presentes en las memorias respectivas una vez que fueran retomados, aunque no explícitamente. Lo que se hizo con las memorias núm. 1, 2 y 3 (éste sólo para la acústica) de cada grupo de alumnos, para un determinado nivel de enseñanza, fue analizar de qué forma se encontraban presentes los aspectos conceptuales referidos.

\section{RESULTADOS}

Los resultados fueron obtenidos analizando la información recogida. Se presentan en tres apartados: lo que se pretendía en términos de desarrollo conceptual (protocolos y ADDCTE), lo que se aprendió efectivamente (tests y memorias) y la manera cómo alumnos y respectivos profesores percibieron la relación entre desarrollo conceptual y TE (opiniones de alumnos y del profesor).

\section{Competencias de utilización de los conceptos a desa- rrollar en los alumnos}

Se identificaron los aspectos conceptuales explícitos e implícitos en el primer protocolo y respectivo ADDCTE de mecánica y acústica. Los de mecánica se encuentran en el cuadro VI, los de acústica en el cuadro VIII. En el caso de la mecánica aun se consideraron los aspectos señalados en el cuadro VII que resultaron del análisis efectivo de las competencias requeridas para responder al test de evaluación de conceptos. Estos aspectos conceptuales, por ser fundamentales, deberían estar presentes y desarrollarse a lo largo de toda la unidad de enseñanza. La hipótesis de la que partimos es que los ADDCTE, al ayudar a los profesores a concretar y encuadrar los aspectos conceptuales de cada actividad experimental, facilitarían que fuesen sucesivamente retomados por los profesores. De esta forma estos aspectos conceptuales deberían estar presentes y desarrollados en otros contextos. Así, diversas competencias de utilización de los conceptos de mecánica o acústica serían desarrolladas en los alumnos.

\section{Análisis de los aprendizajes conceptuales de mecá- nica}

Teniendo en cuenta los aspectos conceptuales identificados (Cuadro VI), se analizaron los aprendizajes efec-

Cuadro VI

Aprendizajes efectuados verificados en las memorias de los TE núm. 1 y 2 de mecánica.

\begin{tabular}{|c|c|c|c|c|c|c|c|}
\hline \multirow[t]{2}{*}{ Aspectos conceptuales } & \multicolumn{7}{|c|}{ Aprendizajes efectuados } \\
\hline & Aa & $\mathbf{A b}$ & Ac & Ad & $\mathbf{B a}$ & $\mathbf{B b}$ & Bc \\
\hline $\begin{array}{l}\text { No es posible conocer el valor exacto de } \\
\text { una magnitud, sólo es posible estimarlo. } \\
\text { Hay siempre un error asociado a una medida. } \\
\text { Forma de estimar el error } \\
\text { asociado a una medida. } \\
\text { Cuantos más valores se obtengan, } \\
\text { más precisa es una medida. }\end{array}$ & $\begin{array}{l}\mathrm{E} / \mathrm{E} \\
\mathrm{E} / \mathrm{E} \\
\mathrm{I} / \mathrm{E}\end{array}$ & $\begin{array}{l}\mathrm{I} / \mathrm{Ic} \\
\mathrm{N} / \mathrm{Ic} \\
\mathrm{I} / \mathrm{Ic}\end{array}$ & $\begin{array}{c}\mathrm{E} / \mathrm{E} \\
\mathrm{Ec} / \mathrm{Ec} \\
\mathrm{Ec} / \mathrm{E}\end{array}$ & $\begin{array}{l}\mathrm{E} / \mathrm{E} \\
\mathrm{E} / \mathrm{Ec} \\
\mathrm{E} / \mathrm{E}\end{array}$ & $\begin{array}{l}\mathrm{Ic} / \mathrm{E} \\
\mathrm{E} / \mathrm{Ec} \\
\mathrm{I} / \mathrm{E}\end{array}$ & $\begin{array}{l}\mathrm{E} / \mathrm{Ec} \\
\mathrm{I} / \mathrm{I} \\
\mathrm{I} / \mathrm{N}\end{array}$ & $\begin{array}{l}\mathrm{E} / \mathrm{I} \\
\mathrm{N} / \mathrm{I} \\
\mathrm{I} / \mathrm{Ic}\end{array}$ \\
\hline $\begin{array}{l}\text { Es necesario conocer la contribución de los } \\
\text { instrumentos de medida para los errores } \\
\text { asociados a una medida. }\end{array}$ & $\mathrm{I} / \mathrm{E}$ & $\mathrm{N} / \mathrm{N}$ & $\mathrm{E} / \mathrm{E}$ & $\mathrm{E} / \mathrm{E}$ & $\mathrm{E} / \mathrm{E}$ & $\mathrm{I} / \mathrm{E}$ & $\mathrm{I} / \mathrm{I}$ \\
\hline $\begin{array}{l}\text { El resultado de una medida debe ser } \\
\text { expresado en términos de un intervalo } \\
\text { de valores: } \\
\text { a) en términos gráficos; } \\
\text { b) en términos matemáticos. }\end{array}$ & $\begin{array}{l}\mathrm{E} / \mathrm{Ec} \\
\mathrm{E} / \mathrm{Ec}\end{array}$ & $\begin{array}{l}\mathrm{Ec} / \mathrm{Ec} \\
\mathrm{N} / \mathrm{N}\end{array}$ & $\begin{array}{l}\mathrm{E} / \mathrm{E} \\
\mathrm{E} / \mathrm{Ec}\end{array}$ & $\begin{array}{l}\mathrm{E} / \mathrm{E} \\
\mathrm{E} / \mathrm{E}\end{array}$ & $\begin{array}{l}\mathrm{E} / \mathrm{E} \\
\mathrm{E} / \mathrm{E}\end{array}$ & $\begin{array}{l}\mathrm{E} / \mathrm{N} \\
\mathrm{E} / \mathrm{N}\end{array}$ & $\begin{array}{l}\mathrm{N} / \mathrm{E} \\
\mathrm{N} / \mathrm{N}\end{array}$ \\
\hline $\begin{array}{l}\text { Los conceptos de precisión y exactitud } \\
\text { se abordan de manera inicial. }\end{array}$ & $\mathrm{N} / \mathrm{I}$ & $\mathrm{N} / \mathrm{N}$ & $\mathrm{I} / \mathrm{I}$ & $\mathrm{I} / \mathrm{I}$ & $\mathrm{N} / \mathrm{N}$ & $\mathrm{N} / \mathrm{N}$ & $\mathrm{N} / \mathrm{N}$ \\
\hline $\begin{array}{l}\text { Existe modelo implícito relativo a la medi- } \\
\text { ción de magnitudes. }\end{array}$ & Ic / I & $\mathrm{N} / \mathrm{N}$ & Ic / Ic & $\mathrm{I} / \mathrm{I}$ & Ic / Ic & Ic / Ic & Ic / Ic \\
\hline
\end{tabular}

Abreviaturas: ... / ... referencia a 1a. memoria / 2a. memoria; Aa: grupo a del profesor A; E: explícito; I: - implícito; Ec o Ic: explícito o implícito en un contexto específico; $N$ : no observado. 
tuados, a partir de las memorias de cada grupo. En primer lugar se consideran las memorias de los TE núm. 1. Después se analizó cómo estaban presentes los mismos aspectos conceptuales en las memorias de los TE núm. 2. Los resultados se encuentran en el cuadro VI.

Sinteticemos la información del cuadro VI:

La imposibilidad de conocer el valor exacto de una medida es una competencia que, en sus aspectos más simples se desarrolló; esto es, los alumnos saben que hay un error asociado a una medida en un contexto ampliado de su utilización. Para los aspectos más operacionales (estimar error y aumentar precisión de una medida), los alumnos del profesor A manifiestan una cierta competencia en los dos contextos utilizados, mientras que los alumnos del profesor B manifiestan deficiencias en los dos contextos. En particular, la forma de estimar un error para medidas directas (TE núm. 1) y sobretodo para medidas indirectas (TE núm. 2) es una competencia muy localizada en los alumnos del profesor A y prácticamente inexistente en los alumnos del profesor B.

La necesidad de conocer la contribución de los instrumentos de medida para errores asociados a una medida es una competencia desarrollada en cualquiera de los TE por la mayoría de los grupos de los dos profesores.

La expresión del resultado de una medida directa (TE núm. 1) fue bien entendida por la generalidad de los grupos, pero para una medida indirecta (TE núm. 2), se encontraron varias dificultades, sobre todo en los alumnos del profesor B.

La mayoría de los grupos interiorizó un modelo, aunque simple, relativo a la medición de magnitudes físicas. El siguiente extracto es ejemplificador:

A lo largo del trabajo experimental se confirmó que existe divergencia entre lo que es exprimido por una fórmula utilizada en la física y los resultados obtenidos y las conclusiones adquiridas de la práctica. Este hecho se debe a innumerables factores, tales como la existencia del error, el estado del material utilizado, entre otros. Veamos el siguiente ejemplo: la fuerza para una deformación de $0 \mathrm{~cm}$ y teóricamente $0 \mathrm{~N}$, pero en el gráfico y por los valores obtenidos se comprobó que la fuerza es $0 \pm 0,05 \mathrm{~N}$.

Un aspecto específico del modelo teórico de la medición de magnitudes físicas como los conceptos de precisión y exactitud y sus relaciones aparece muy incipiente, tal vez porque ninguno de los profesores le dio importancia.

Otro aspecto importante es el aprendizaje efectuado con ciertos conceptos que puede ser analizado a partir de las respuestas a los pretest y los postests. Se escogieron cinco alumnos, al azar. Se presentan los resultados en el cuadro VII relativos a los aprendizajes efectuados, donde los aspectos conceptuales en juego están explicados en la columna respectiva.

Cuadro VII

Análisis de respuestas de los alumnos al pretest y al postest.

\begin{tabular}{|c|c|c|c|c|c|c|c|c|c|c|}
\hline \multirow[t]{2}{*}{ Aspectos conceptuales } & \multicolumn{10}{|c|}{ Aprendizajes efectuados } \\
\hline & Aa & $\mathbf{A b}$ & Ac & Ad & Ae & $\mathbf{B a}$ & $\mathbf{B b}$ & Bc & Bd & $\mathbf{B e}$ \\
\hline $\begin{array}{l}\text { El cálculo del trabajo hecho a partir de } \\
\text { magnitudes medidas no es exacto. }\end{array}$ & $\mathrm{N} / \mathrm{S}$ & $\mathrm{N} / \mathrm{N}$ & $\mathrm{N} / S$ & $-/ S$ & $\mathrm{~N} / \mathrm{S}$ & $\mathrm{N} / \mathrm{N}$ & $\mathrm{N} / \mathrm{N}$ & $\mathrm{N} / \mathrm{N}$ & $\mathrm{N} / \mathrm{N}$ & $\mathrm{N} / \mathrm{N}$ \\
\hline $\begin{array}{l}\text { Cálculo, de forma adecuada a la situación, } \\
\text { del trabajo realizado por determinadas fuerzas. }\end{array}$ & $\mathrm{N} / \mathrm{S}$ & $\mathrm{N} / \mathrm{S}$ & $\mathrm{N} / \mathrm{N}$ & $\mathrm{N} / \mathrm{S}$ & $\mathrm{N} / \mathrm{N}$ & $-/ S$ & $-/ S$ & $\mathrm{~N} / \mathrm{S}$ & $\mathrm{N} / \mathrm{S}$ & $\mathrm{S} / \mathrm{S}$ \\
\hline $\begin{array}{l}\text { Análisis de la conservación de la energía } \\
\text { (en una situación física descrita de } \\
\text { forma realista) para responder a una cuestión } \\
\text { fuertemente contextualizada: se recurre } \\
\text { a un modelo ideal o a un modelo más realista. }\end{array}$ & $\mathrm{Ie} / \mathrm{Ic}$ & $\begin{array}{l}\mathrm{Rc} / \\
\mathrm{Rc}\end{array}$ & $\begin{array}{l}\mathrm{CA} / \\
\mathrm{CA}\end{array}$ & $\mathrm{Ic} / \mathrm{Ic}$ & $\mathrm{Ic} / \mathrm{Ic}$ & $\begin{array}{l}\text { CA/ } \\
\text { Re }\end{array}$ & $\begin{array}{l}\mathrm{Rc} / \\
\mathrm{Rc}\end{array}$ & $\begin{array}{l}\text { CA/ } \\
\text { CA }\end{array}$ & $\mathrm{Re} / \mathrm{Ic}$ & $\mathrm{Ie} / \mathrm{Rc}$ \\
\hline $\begin{array}{l}\text { Análisis de la conservación de la energía } \\
\text { (en la misma situación física descrita de } \\
\text { forma realista) para que respondan a la } \\
\text { cuestión general: se recurre a un modelo } \\
\text { ideal o a un modelo más realista. }\end{array}$ & $\begin{array}{l}\mathrm{Rc} / \\
\mathrm{Rc}\end{array}$ & $\begin{array}{l}\mathrm{CA} / \\
\mathrm{CA}\end{array}$ & $\begin{array}{l}\mathrm{Re} / \\
\mathrm{Rc}\end{array}$ & $\begin{array}{l}\text { CA/ } \\
\text { Re }\end{array}$ & $\begin{array}{l}\mathrm{CA} / \\
\mathrm{Rc}\end{array}$ & $-/ \mathrm{Ie}$ & $\mathrm{Ie} / \mathrm{Ie}$ & $-/ \mathrm{Rc}$ & $\mathrm{Ie} / \mathrm{Rc}$ & $\begin{array}{l}\mathrm{CA} / \\
\mathrm{CA}\end{array}$ \\
\hline $\begin{array}{l}\text { En aspectos operacionales de análisis de } \\
\text { transferencia de energía razonan o calculan } \\
\text { correctamente. }\end{array}$ & $\mathrm{N} / \mathrm{S}$ & $\mathrm{N} / \mathrm{N}$ & $\mathrm{N} / \mathrm{S}$ & $\mathrm{N} / \mathrm{S}$ & $\mathrm{N} / \mathrm{S}$ & $-/-$ & $-/-$ & $-1-$ & $\mathrm{N} / \mathrm{S}$ & $\mathrm{N} / \mathrm{S}$ \\
\hline
\end{tabular}

Abreviaturas: ....... - referencia a pretest / postest; Aa: alumno a del profesor A; $N$ : no ; S: sí; Ic o Ie: modelo ideal con justificación correcta o no correcta; Rc o Re: modelo realista con justificación correcta o no correcta; $C A$ : concepción alternativa; - no se aplica. 
Cuadro VII

Aprendizajes efectuados verificados en las memorias de los TE núm. 1, 2 y 3 de acústica.

\begin{tabular}{|c|c|c|c|c|c|c|c|c|}
\hline \multirow[t]{2}{*}{ Aspectos conceptuales } & \multicolumn{8}{|c|}{ Aprendizajes efectuados } \\
\hline & $\mathrm{Ca}$ & $\mathrm{Cb}$ & $\mathrm{Cc}$ & $\mathrm{Cd}$ & $\mathrm{Da}$ & $\mathrm{Db}$ & $\mathrm{Dc}$ & Dd \\
\hline $\begin{array}{l}\text { Diferencia de sonidos por: } \\
\text { altura } \\
\text { intensidad }\end{array}$ & $\begin{array}{l}\mathrm{S} / \mathrm{S} / \mathrm{S} \\
\mathrm{S} / \mathrm{S} /-\end{array}$ & $\begin{array}{l}\mathrm{S} / \mathrm{S} / \mathrm{S} \\
\mathrm{S} / \mathrm{S} /-\end{array}$ & $\begin{array}{l}\mathrm{S} / \mathrm{S} / \mathrm{S} \\
\mathrm{S} / \mathrm{S} /-\end{array}$ & $\begin{array}{l}\mathrm{N} / \mathrm{S} /- \\
\mathrm{N} / \mathrm{S} /-\end{array}$ & $\begin{array}{l}\mathrm{N} / \mathrm{S} / \mathrm{S} \\
\mathrm{S} / \mathrm{S} /-\end{array}$ & $\begin{array}{l}-/ \mathrm{S} / \mathrm{S} \\
\mathrm{S} / \mathrm{S} /-\end{array}$ & $\begin{array}{l}\mathrm{S} / \mathrm{S} / \mathrm{S} \\
\mathrm{S} / \mathrm{S} /-\end{array}$ & $\begin{array}{l}-/ \mathrm{S} /- \\
\mathrm{S} / \mathrm{S} /-\end{array}$ \\
\hline $\begin{array}{l}\text { Describe sonidos por: } \\
\text { altura } \\
\text { intensidad }\end{array}$ & $\begin{array}{l}\mathrm{N} / \mathrm{S} / \mathrm{S} \\
\mathrm{S} / \mathrm{S} /-\end{array}$ & $\begin{array}{l}\mathrm{N} / \mathrm{S} / \mathrm{S} \\
\mathrm{S} / \mathrm{S} / \mathrm{S}\end{array}$ & $\begin{array}{l}\mathrm{S} / \mathrm{S} / \mathrm{S} \\
\mathrm{S} / \mathrm{S} / \mathrm{S}\end{array}$ & $\begin{array}{l}\mathrm{S} / \mathrm{S} /- \\
\mathrm{N} / \mathrm{S} /-\end{array}$ & $\begin{array}{l}\mathrm{N} / \mathrm{S} / \mathrm{S} \\
\mathrm{N} / \mathrm{S} / \mathrm{S}\end{array}$ & $\begin{array}{l}-/ N / S \\
S / N / N\end{array}$ & $\begin{array}{l}\mathrm{N} / \mathrm{N} / \mathrm{S} \\
\mathrm{N} / \mathrm{S} / \mathrm{N}\end{array}$ & $\begin{array}{l}\mathrm{S} / \mathrm{N} /- \\
\mathrm{S} / \mathrm{S} /-\end{array}$ \\
\hline $\begin{array}{l}\text { Produce sonidos con determinadas } \\
\text { características de } \\
\text { Altura } \\
\text { Intensidad }\end{array}$ & $\begin{array}{l}\mathrm{S} / \mathrm{S} / \mathrm{S} \\
\mathrm{S} / \mathrm{S} / \mathrm{S}\end{array}$ & $\begin{array}{l}\mathrm{S} / \mathrm{S} / \mathrm{S} \\
\mathrm{S} / \mathrm{S} / \mathrm{S}\end{array}$ & $\begin{array}{l}\mathrm{S} / \mathrm{S} / \mathrm{S} \\
\mathrm{S} / \mathrm{S} / \mathrm{S}\end{array}$ & $\begin{array}{l}\mathrm{S} / \mathrm{S} / \mathrm{S} \\
\mathrm{S} / \mathrm{S} / \mathrm{S}\end{array}$ & $\begin{array}{l}\mathrm{S} /-/- \\
\mathrm{S} / \mathrm{S} /-\end{array}$ & $\begin{array}{l}-1-1- \\
\mathrm{S} /-1-\end{array}$ & $\begin{array}{l}\mathrm{S} /-/- \\
\mathrm{S} /-/-\end{array}$ & $\begin{array}{l}\mathrm{S} /-/- \\
\mathrm{S} / \mathrm{S} /-\end{array}$ \\
\hline $\begin{array}{l}\text { Relaciona correctamente altura } \\
\text { y intensidad de diferentes sonidos }\end{array}$ & $\mathrm{S} / \mathrm{S} / \mathrm{S}$ & $\mathrm{N} / \mathrm{S} / \mathrm{S}$ & $\mathrm{N} / \mathrm{S} / \mathrm{S}$ & N / S / - & N / N / - & $\mathrm{Sc} / \mathrm{N} /-$ & $\mathrm{Sc} / \mathrm{N} /-$ & $\mathrm{S} / \mathrm{N} /-$ \\
\hline $\begin{array}{l}\text { Utiliza un modelo teórico relativo } \\
\text { a la producción y transmisión } \\
\text { de sonidos: } \\
\text { Asocia sonidos a vibraciones } \\
\text { del medio } \\
\text { Transmisión de vibraciones de un } \\
\text { medio a otro } \\
\text { Representa o reconoce } \\
\text { representación del sonido por onda }\end{array}$ & $\begin{array}{l}\mathrm{S} / \mathrm{S} / \mathrm{-} \\
-/ \mathrm{S} /- \\
-/ \mathrm{Sc} / \mathrm{Sc}\end{array}$ & $\begin{array}{l}\mathrm{S} / \mathrm{S} / \mathrm{-} \\
-/ \mathrm{Sc} /- \\
-/ \mathrm{Sc} / \mathrm{Sc}\end{array}$ & $\begin{array}{l}-/ \mathrm{S} /- \\
-/ \mathrm{S} /- \\
-/ \mathrm{Sc} / \mathrm{Sc}\end{array}$ & $\begin{array}{l}\mathrm{S} / \mathrm{S} /- \\
-/ \mathrm{Sc} /- \\
-/ \mathrm{N} / \mathrm{S}\end{array}$ & $\begin{array}{l}\mathrm{Sc} / \mathrm{S} / \text { - } \\
-/ \mathrm{Sc} /- \\
-/ \mathrm{Sc} / \mathrm{Sc}\end{array}$ & $\begin{array}{l}\mathrm{N} / \mathrm{S} /- \\
-/-/- \\
-/ \mathrm{N} / \mathrm{Sc}\end{array}$ & $\begin{array}{l}-/ \mathrm{S} /- \\
-/ \mathrm{Sc} /- \\
-/ \mathrm{N} / \mathrm{Sc}\end{array}$ & $\begin{array}{l}\mathrm{N} / \mathrm{S} /- \\
-/ \mathrm{Sc} /- \\
-/ \mathrm{Sc} /-\end{array}$ \\
\hline
\end{tabular}

Abreviaturas: .......... referencia a 1a. memoria / 2a. memoria / 3a. memoria; Aa: grupo a del profesor $A ; S$ o $N$ : sí o no; $S c$ : sí en un contexto específico; -: no responde, o no se puede clasificar, o no se aplica.

La información del cuadro VII puede ser sintetizada del siguiente modo:

La cuestión de saber si el cálculo del trabajo efectuado a partir de magnitudes medidas daría o no un valor exacto permite evidenciar dificultades relativas a las magnitudes indirectas ya manifestadas en las memorias, sobretodo para los grupos del profesor B.

El cálculo del trabajo de forma adecuada a la situación física en el postest es una ventaja, en términos de aprendizaje, que es doble: adquisición de nuevas relaciones entre conceptos y flexibilidad del raciocinio.

Igualmente, la adquisición de invariantes para el análisis y cálculo de casos de transferencia de energía, en situaciones específicas, es un aspecto del desarrollo conceptual verificado en la confrontación entre el pretest y el postest.

La utilización del modelo teórico de la conservación de la energía necesita modelizarse en situaciones físicas específicas para que pueda utilizarse correctamente. El modelo de la situación física puede ser ideal o más realista, aunque este último sea más adecuado a la propia situación física. El análisis de las respuestas a los tests de evaluación de conceptos indica, para una cuestión fuer- temente contextualizada, que los alumnos del profesor A recurren tendencialmente a un modelo ideal de la situación física y no se verifica una evolución desde el pretest hasta el postest. Los alumnos del profesor B presentan mayor variabilidad en el tipo de modelización de la situación física que efectuaron y tampoco se verifica, en general, evolución del pretest hasta el postest. Si la cuestión es general, los alumnos del profesor A recurren, al final de la unidad curricular, a un modelo de la situación física más realista y ultrapasan algunas concepciones alternativas evidenciando así una evolución favorable del pretest hacia el postest. En los alumnos del profesor B, la variabilidad de las respuestas es mayor y no se nota una tendencia evolutiva clara, en particular no ultrapasando concepciones alternativas.

\section{Análisis de los aprendizajes conceptuales de acústica}

Los aprendizajes efectuados por los alumnos referentes a la acústica están en el cuadro VIII y se sintetizan como se explica a continuación:

Los alumnos del profesor C, en general, diferencian, describen y producen sonidos atendiendo a su altura o a su intensidad en contextos y tareas diferentes. Para los alumnos del profesor $\mathrm{D}$, sólo pasa lo mismo con relación 
Cuadro IX

Síntesis de las opiniones de los alumnos sobre la importancia de los trabajos experimentales para relacionar los conceptos.

\begin{tabular}{|c|c|c|}
\hline Alumnos & Respuesta & Justificación \\
\hline \multirow[t]{2}{*}{ Profesor A } & Importante & $\begin{array}{l}\text { Posibilita mayor comprensión-aprendizaje de los aspectos teóricos } \quad(16 / 26) \\
\text { Promueve mayor interés } \quad(4 / 26)\end{array}$ \\
\hline & No importante & $\begin{array}{ll}\text { Falta de interés o atención } & (3 / 26) \\
\text { Dificultad en los conceptos } & (3 / 26)\end{array}$ \\
\hline \multirow[t]{2}{*}{ Profesor B } & Importante & Posibilita mayor comprensión o aprendizaje de los aspectos teóricos (13/20) \\
\hline & No importante & Prefieren el método tradicional (ejercicios y exposición del profesor) $(7 / 20)$ \\
\hline Profesor C & Importante & $\begin{array}{l}\text { Comprensión de fenómenos } \quad(18 / 26) \\
\text { Para distinguir conceptos específicos }(11 / 26) \\
\text { Comprensión de la propagación del sonido }(18 / 26) \\
\text { Conocimiento de fenómenos nuevos } \quad(12 / 26)\end{array}$ \\
\hline Profesor D & Importante & $\begin{array}{l}\text { Comprensión de fenómenos }(11 / 20) \\
\text { Comprensión de la propagación del sonido }(11 / 20) \\
\text { Conocimiento de fenómenos nuevos }(18 / 20)\end{array}$ \\
\hline
\end{tabular}

a la diferenciación de los sonidos. Algunos grupos de este profesor describen bien los sonidos después del TE núm. 1 (grupo a) y los restantes grupos no utilizan simultáneamente las dos características (altura e intensidad). La producción de sonidos en los alumnos del profesor D está menos desarrollada que en los alumnos del profesor $\mathrm{C}$ y más restringida a las situaciones del TE núm. 1 .

Cuando se trata de relacionar la altura y la intensidad de diferentes sonidos, los alumnos del profesor $\mathrm{C}$ tienen, en general, más éxito a través de los TE subsecuentes al núm. 1. Por el contrario, los alumnos del profesor D no relacionan, en general, las dos características.

Con relación a los diferentes aspectos de un modelo teórico de producción y transmisión de sonidos, se veri- fica que solamente la asociación de sonidos a vibraciones del medio es apropiada por todos los grupos de los dos profesores. A medida que el aspecto en causa del modelo teórico es más abstracto, más localizada es la competencia y más tarde se manifiesta. Este aspecto está más marcado en los alumnos del profesor D. Todavía, la mayoría de los grupos interiorizó algunos aspectos del modelo teórico, relativo a la producción y transmisión de sonidos, aunque en contextos específicos que permitieron a los alumnos observar nuevas experiencias con otras herramientas descriptivas y teóricas, concretamente haciendo la relación entre las interpretaciones macroscópica y microscópica. Los siguientes extractos son ejemplificativos:

[...] las partículas sufrieron un mayor o menor alejamiento de su punto de equilibrio.

Cuadro X

Opiniones de los profesores sobre la importancia de los ADDCTE.

\begin{tabular}{|c|c|c|c|}
\hline Profesor A & Profesor B & Profesor C & Profesor D \\
\hline $\begin{array}{l}\text { Permiten orientar el TE de for- } \\
\text { ma más adecuada. } \\
\text { Enriqueció la discusión pro- } \\
\text { movida con los alumnos en el } \\
\text { sentido de lograr los objetivos } \\
\text { pretendidos con el respectivo } \\
\text { TE. }\end{array}$ & $\begin{array}{l}\text { Permiten tener una visión glo- } \\
\text { bal sobre la relación de los con- } \\
\text { ceptos a trabajar en el transcur- } \\
\text { so de las diferentes tareas a } \\
\text { realizar. } \\
\text { Establecen una relación estre- } \\
\text { cha entre el modelo conceptual } \\
\text { y las competencias a desarrollar } \\
\text { en los alumnos relacionados con } \\
\text { las tareas. } \\
\text { Le permitió incluso al profesor } \\
\text { proporcionar ayudas a los alum- } \\
\text { nos de modo más articulado con } \\
\text { los contenidos, conceptos y ob- } \\
\text { jetivos. }\end{array}$ & $\begin{array}{l}\text { Permiten trabajo de reflexión y } \\
\text { revisión en la fase de } \\
\text { planificación de las clases (y } \\
\text { delos ADDCTE), lo que implica } \\
\text { tomar conciencia de lo que se } \\
\text { está haciendo, cómo se hace, } \\
\text { por qué y para qué se hace. } \\
\text { El modo cómo son utilizados } \\
\text { en el aula depende, pero no de } \\
\text { forma unívoca, del grado de } \\
\text { apropiación conseguido en la } \\
\text { fase de elaboración del } \\
\text { ADDCTE. }\end{array}$ & $\begin{array}{l}\text { Permiten la explicación del } \\
\text { conocimiento científico y di- } \\
\text { dáctico, en dialéctica. } \\
\text { Ayudan a proporcionar un ver- } \\
\text { dadero desarrollo conceptual. } \\
\text { Ayudó el profesor a dejar que } \\
\text { los alumnos verbalizacen su } \\
\text { conocimiento exponiendo y ar- } \\
\text { gumentando sus tesis explica- } \\
\text { tivas. }\end{array}$ \\
\hline
\end{tabular}


El diapasón más corto hizo que el péndulo se moviese deprisa, a menor distancia del diapasón.

El diapasón «más corto» que emitió el sonido más alto emitió vibraciones más rápidas, en relación con el diapasón más largo, en el mismo intervalo de tiempo.

\section{Análisis de las opiniones de alumnos y profesores}

Se pidió a los alumnos de los profesores A, B, C y D que elaborasen un pequeño texto (en casa) y que respondiesen, de forma fundamentada, a la cuestión: «Los trabajos prácticos experimentales, ¿te han ayudado a relacionar los conceptos explicados en las clases teóricas y teóricoprácticas?» Las respuestas están sintetizadas en el cuadro IX.

La opinión de los profesores sobre la importancia de los ADDCTE para el desarrollo conceptual, la orientación de las clases de trabajo experimental y la articulación con las actividades de las restantes clases está presentada en el cuadro X. Fundamentalmente los profesores consideran que los ADDCTE son herramientas que proporcionan: reflexión sobre los conceptos y explicitud de sus particularidades; una gestión articulada y flexible de las actividades experimentales y de las otras actividades en el aula con vistas al desarrollo conceptual.

\section{DISCUSIÓN Y CONCLUSIONES}

Empecemos discutiendo los aprendizajes conceptuales efectuados en acústica. A partir de los resultados está más claro que existen cuatro tipos de competencias cuyo nivel es gradualmente mayor: competencias descriptivas (diferencia... describe...); competencias descriptivo-manipulativas (produce...); competencias para utilizar relaciones entre conceptos en situaciones específicas (relaciona...); competencias para utilizar un modelo teórico en situaciones específicas.

Existen esencialmente dos aspectos a señalar:

a) Las competencias descriptivas son aquéllas que más rápida y fácilmente son desarrolladas y están menos sujetas a variaciones entre grupos. Por el contrario, las competencias de más alto nivel se desarrollan más lentamente y están sujetas a mayores variaciones entre los alumnos de los dos profesores e incluso entre grupos del mismo profesor. El mayor tiempo dedicado por el profesor $\mathrm{C}$, ya sea al trabajo experimental, ya sea a las restantes actividades de enseñanza de la acústica, puede explicar, en parte, el mejor desarrollo, en sus alumnos, de competencias descriptivas y sobretodo de las competencias de más alto nivel. También las percepciones más superficiales, en general, de los alumnos del profesor D sobre el papel de los TE para relacionar conceptos pueden explicar el menor desarrollo de competencias de más alto nivel en estos alumnos. Además de eso, la variabilidad del desarrollo de las competencias de alto nivel debe estar relacionada con la complejidad de la evolución conceptual (Silva, 1999). La utilización de los
ADDCTE por los profesores C y D tiene variaciones importantes. Para el profesor D, los ADDCTE permiten esencialmente la articulación entre asuntos tratados en las diferentes clases. Para el profesor C, además del aspecto referido, hay esencialmente un énfasis en la flexibilización de la gestión del TE en la clase. También este aspecto puede contribuir al mayor desarrollo de las competencias de alto nivel en los alumnos del profesor C.

b) Las competencias descriptivo-manipulativas están claramente más desarrolladas en los alumnos del profesor $\mathrm{C}$ que en los alumnos del profesor D. Las características de los alumnos del profesor D (desinterés, inmediatas) y la forma cómo este profesor utilizó los ADDCTE (no centrado en las actividades experimentales) pueden haber contribuido a que estas competencias no se hubiesen desarrollado convenientemente en sus alumnos. $\mathrm{Pa}-$ rece, sin embargo, que hay una relación entre el mayor desarrollo de esta competencia y el desarrollo de las competencias de más alto nivel (relacionar conceptos y utilizar modelos). Aparentemente la acción bien conseguida de los sujetos sobre situaciones físicas concomitantemente a otros aspectos es condición necesaria para que los conceptos se relacionen y se utilicen modelos teóricos en situaciones físicas diversificadas. Este aspecto está claramente de acuerdo con el cuadro teórico. De él resalta que la acción del sujeto tiene que orientarse de manera que se adecúe a la situación física y a la herramienta conceptual utilizada y tiene que estar articulada con las restantes dimensiones de construcción y utilización de los conceptos.

Los resultados relativos a los aprendizajes de mecánica nos permiten evidenciar diversos tipos de competencias en la utilización de conceptos: competencias operacionales de bajo nivel del modelo teórico de medición de magnitudes físicas (reconocimiento de que hay siempre errores, expresión del resultado, contribución del aparato de medida...); competencias de alto nivel del modelo teórico de medición de magnitudes físicas (estimar el error, hacer más precisa una medida); competencias de alto nivel del modelo teórico de medición de magnitudes físicas como un todo; competencias relativas a la utilización de conceptos de trabajo y transferencias de energía, en situaciones físicas específicas; competencias relativas a la construcción de modelos de situaciones físicas adecuados a la utilización del modelo teórico de la conservación de la energía.

Los aspectos a señalar son esencialmente dos:

a) Tal como se ha visto para la acústica, también en la mecánica, en el nivel de enseñanza considerado, las competencias de bajo nivel son más rápida y fácilmente desarrolladas, estando menos sujetas a variación entre grupos de alumnos. Las competencias relativas a la utilización de conceptos centrales de la mecánica aparecen bien desarrolladas al final de la unidad de enseñanza en contextos en los que las situaciones físicas se describen de forma realista. Es de suponer que los ADDCTE, concretando los conceptos y modelos teóricos y relacionándolos, hayan ayudado a los profesores a enseñar más eficazmente estos aspectos. Las competencias de alto 
nivel, tanto en lo que le concierne al modelo teórico de medición de magnitudes como a la construcción de modelos de situaciones físicas específicas, están menos desarrollados, sobretodo en los alumnos del profesor B, y presentan mayor variabilidad entre alumnos. Muchos alumnos del profesor B prefieren ejercicios y la exposición del profesor en vez de actividades experimentales articuladas con otras clases, ya que saben que en los exámenes estos aspectos no se valorizan y, por otro lado, quieren conseguir buenas notas. Este hecho explica, en parte, el poco desarrollo de estas competencias en estos alumnos. Para el profesor A, el ADDCTE permite centrar su atención en el trabajo experimental, mientras, que para el profesor B, el papel de los ADDCTE es más de articulación entre los diferentes tipos de actividades realizadas en las clases. El profesor B también ocupa menos tiempo con la realización de los TE. Estos dos factores pueden explicar, también, el menor desarrollo de las competencias de alto nivel en los alumnos del profesor B.

b) No hay dudas de que los alumnos recurren a diferentes modelos de la situación física conforme la cuestión sea contextualizada o general. Como hay evolución en el sentido de utilizar modelos más realistas de situaciones físicas en las respuestas a una de las cuestiones, parece haber una preocupación de los profesores de este estudio en introducir esta forma de razonar. Los ADDCTE, probablemente, ayudaron a los profesores en esta tarea porque concretan los modelos teóricos y las particularidades de la situación física. Puede haber dos explicaciones igualmente válidas, que sería necesario profundizar con otros estudios. Los alumnos comprenden las cuestiones contextualizadas como académicas y utilizan modelos que, desde su punto de vista, garantizan éxito escolar o la competencia de construir modelos de la situación física más realistas. Responder a cuestiones contextualizadas es bastante difícil de desarrollar y una unidad curricular es insuficiente para permitirlo.

Existen constreñimientos en la propia implantación de las actividades experimentales. Los principales son el mayor o menor interés de los alumnos por las actividades experimentales (ya que éstas no son automáticamente motivadoras [Hodson, 1994]) y las condiciones logísticas de funcionamiento y utilización del laboratorio. Están igualmente marcadas por la dificultad de articulación entre actividades experimentales y aspectos teóricos (más difícil durante el $10^{\circ}$ curso). Existen igualmente constreñimientos en la utilización de los ADDCTE, pues la articulación entre aspectos experimentales y aspectos teóricos es muy difícil debido a la tradición de enseñanza que las separa de forma bien marcada.

De la discusión de los resultados del aprendizaje relativos a la acústica se puede concluir que el desarrollo de competencias en la construcción y la utilización de conceptos físicos, a través del trabajo experimental, depende de varios factores: tiempo de aprendizaje, forma como los TE se gestionaron en la clase, percepción de los alumnos en lo que se refiere al propio papel de los TE, nivel de las propias competencias, entre otros. Los resultados de aprendizaje relativos a la mecánica confirman la importancia de estos factores, con excepción del tiempo de aprendizaje.

Sobre todo a partir de los resultados relativos a la acústica y su discusión se puede apuntar, por lo menos como hipótesis de trabajo a confirmar con otros estudios, que el desarrollo de competencias de alto nivel en la utilización de conceptos en situaciones diversificadas parece depender de tres factores: $a$ ) tiempo de aprendizaje; $b$ ) forma cómo los ADDCTE fueron utilizados; c) desarrollo previo y concomitante de competencias descriptivo-manipulativas. El factor $c$ puede, además, estar relacionado con el modo de utilización de los ADDCTE. La importancia específica del desarrollo de competencias descritivo-manipulativas para el desarrollo de competencias de alto nivel no pudo estudiarse en la mecánica. Además, su importancia justificaría un estudio por sí solo.

La tendencia de la enseñanza de la física de utilizar modelos ideales no explicitados en un determinado contexto específico induce a un tratamiento superficial de situaciones físicas que recurre a modelos ideales sin explicar las restricciones asumidas de cara a la situación física particular. La actividad experimental apoyada en los ADDCTE puede contrariar esta tendencia, en la medida en que explicitan los modelos físicos subyacentes a las situaciones físicas, así como sus particularidades y las condiciones de utilización de los modelos teóricos. Además, la competencia de construir modelos de situaciones físicas parece ser una competencia que carece de más tiempo de desarrollo y puede necesitar enfoques que no se agoten en las actividades experimentales.

No es posible comparar directamente los resultados de aprendizaje de los alumnos de los profesores A y B con los resultados de aprendizaje de los alumnos de los profesores $\mathrm{C}$ y $\mathrm{D}$ porque son sobre contenidos y niveles de enseñanza diferentes. Sin embargo, existen cuatro aspectos comunes a los profesores A y $\mathrm{C}$ y a los profesores B y D. Los profesores A y C, al contrario de los profesores B y D: a) emplearon más tiempo con las actividades experimentales; y $b$ ) utilizaron los ADDCTE de forma que centrasen su atención en los TE y en el modo en cómo los explotaban conceptualmente. Sus alumnos, al contrario de los alumnos de los profesores B y D: c) desarrollaron, comparativamente, competencias del más alto nivel; y $d$ ) presentaron opiniones más favorables o menos superficiales sobre la importancia del TE.

Parece que hay, así, una relación fuerte entre, por un lado, la mayor inversión en el TE en términos de tiempo y la forma cómo se utilizaron los ADDCTE ayudando al profesor a centrarse más en el propio TE; y, por otro, entre el mayor desarrollo en los alumnos de competencias de alto nivel y mejores expectativas en relación con el TE. Los ADDCTE tuvieron un papel importante para que en algunos profesores tuviese lugar un mayor énfasis en la forma cómo el trabajo experimental se ejecuta, se interpreta y profundiza sus aspectos conceptuales. El mayor énfasis en el propio trabajo experimental articulando ejecución de las actividades experimentales con la interpretación y profundización de sus aspectos 
conceptuales (conceptos, relaciones, operaciones, particularidades, modelos de las situaciones físicas y modelos teóricos, entre otros) puede ser una forma de operar, en la práctica lectiva, los tres componentes del cuadro teórico: a) el aprendizaje conceptual se apoya en la acción del sujeto en articulación con la utilización de lenguaje para comunicar y operar sobre entidades conceptuales y sus relaciones; $b$ ) el aprendizaje de los conceptos físicos es evolutivo; y c) el profesor tiene un papel determinante en la mediación de los aprendizajes. El énfasis referido es un modo de efectuar interacciones entre ideas concomitantemente con las interacciones con lo dispositivo experimental. Como se ve, puede ser determinante para el desarrollo de competencias de alto nivel y es, ciertamente, una forma de juntar el cómo acontece al porqué acontece (Wellington, 1994), de aumentar el nivel de conceptualización a través de la interacción entre la realidad y su modelización (Giuseppin, 1996) y de «reconstruir» las ideas de los alumnos (Gunstone, 1991).

A pesar de los constreñimientos referidos anteriormente - de cara a la forma cómo los ADDCTE fueron concebidos (por los profesores y articulados con los protocolos experimentales) y utilizados en la clase, y a los resultados del análisis de los aprendizajes-, parece ser posible afirmar que el recurso didáctico ADDCTE, siempre que sea apropiado por el profesor, puede contribuir en el desarrollo de competencias elementales y no elementales en variadas vertientes de los campos conceptuales en estudio: mecánica y acústica. En concreto permitió construir, para la enseñanza básica, herramientas de descripción de los sonidos, lo que es particularmente notable para la unidad y nivel de enseñanza en causa y, para la enseñanza secundaria, la utilización de un modelo simple sobre la medición de magnitudes físicas. Existen algunas competencias, sobretodo las de alto nivel, que necesitan ser objeto de atención especial, variando según el grupo de alumnos, lo que está de acuerdo con resultados de investigación que apuntan a la complejidad del desarrollo conceptual (Silva, 1999).

\section{REFERENCIAS BIBLIOGRÁFICAS}

DUMAS-CARRÉ, A. y WEIL-BARAIS, A. (1998). Tutelle et médiation dans l'éducation scientifique. Berna: Peter Lang.

FRANCESCHELLI, S. y WEIL-BARAIS, A. (1998). La routine conversationnelle comme stratégie de changement conceptuel: apprendre à modéliser en mécanique, en DumasCarré, A. y Weil-Barais, A. (eds.). Tutelle et médiation dans l'éducation scientifique, pp. 211-238. Berna: Peter Lang.

GARCÍA, J.J., PRO, A. y SAURA, O. (1995). Planificación de una unidad didáctica: el estudio del movimiento. Enseñanza de las Ciencias, 13(2), pp. 211-226.
Todos los profesores del estudio participaron en la elaboración de los ADDCTE y, sin embargo, no los utilizaron de la misma manera ni valoraron de igual modo el trabajo experimental. Esta constatación nos remite a dos tipos de afirmaciones. Primero, atendiendo a que una de las funciones de los ADDCTE, reconocidas por los profesores, es la de que ayudan a preparar didáctica y científicamente los asuntos a enseñar y las competencias a desarrollar, será deseable que los profesores participen en su elaboración. Segundo, independientemente de las conclusiones que se pudieron sacar del estudio, se plantean dos tipos de cuestiones: $a$ ) El énfasis dado, o no, a las actividades experimentales y respectivos aspectos conceptuales, ¿está relacionado, con profundidad, con las concepciones de los profesores acerca de las actividades experimentales y de su papel en el aprendizaje? b) El conocimiento físico que los profesores tienen que movilizar en la explotación de las actividades experimentales, ¿inhibe a aquéllos que no están seguros de sus competencias en la utilización del mismo conocimiento físico?

De cara a los resultados disponibles y a las limitaciones del estudio, la respuesta a nuestro problema de investigación es sólo indicativa. Parece, sin embargo, que es posible desarrollar competencias de diferentes niveles utilizando actividades experimentales apoyadas por auxiliares didácticos que denominamos auxiliares didácticos del desarrollo conceptual a través del trabajo experimental (ADDCTE). No obstante, para el desarrollo de competencias de alto nivel, el TE tiene que ser efectivamente valorizado en términos de tiempo y los ADDCTE, una vez apropiados por los docentes, tienen que ser utilizados para mejor concretar, interpretar y profundizar los aspectos conceptuales inherentes a la actividad experimental. Parece importante articular las diversas actividades de enseñanza. Parece aún más importante concretar y articular los aspectos conceptuales propios de la actividad experimental en estrecha relación con la manipulación experimental propiamente dicha.
GIUSEPPIN, M. (1996). Place et rôle des activités expérimentales en sciences physiques. Didaskalia, 9, pp. 107-118.

GUNSTONE, R. (1991). Reconstructing theory from practical experience, en Woolnough, B. (ed.). Practical Science, pp. 67-77. Milton Keynes: Open University Press

HODSON, D. (1994). Hacia un enfoque más crítico del trabajo de laboratorio. Enseñanza de las Ciencias, 12(3), pp. 299-313.

JOURNEAUX, R. (1996). La référence au réel: les sciences physiques et les recours à la mesure, en Toussaint, J. Didactique 
appliquée de la physique-chimie, pp. 179-194. París: Nathan.

LARCHER, C. (1996). La physique et la chimie, science de modèles, en Toussaint, J. Didactique appliquée de la physiquechimie, pp. 160-178. París: Nathan.

LEMEIGNAN, G. y WEIL-BARAIS, A. (1993). Construire des concepts en Physique. París: Hachette.

LOPES, J.B. (1999). «Avaliação multidimensional de campos conceptuais de Mecânica. Papel da modelização na aprendizagem». Tesis doctoral (inédita). Vila Real: Universidad de Trás-os-Montes e Alto Douro.

LOPES, J.B., COELHO, A., FORTUNA, A., OLIVEIRA, A., SILVA, J., CARRILHO, L. y SILVA, M. (2000). Promover o desenvolvimento conceptual em Física através do trabalho experimental. Vila Real: Universidad de Trás-os-Montes e Alto Douro.

LOPES, J.B., COSTA, N., WEIL-BARAIS, A. y DUMASCARRÉ, A. (1999). Évaluation de la maîtrise des concepts de la mécanique chez des étudiants et des professeurs. Didaskalia, 14, pp. 11-38.

MARTINAND, J.L. (1995). Introduction à la modélisation, en Martinand, J.L. y Durey, A. (eds.). Actes Séminaire de Didactique des disciplines technologiques, pp. 126-138. Cachan: Association Tour 123.

PRO BUENO, A. (1999). Planificación de unidades didácticas por los profesores: análisis de tipos de actividades de enseñanza. Enseñanza de las Ciencias, 17 (3), pp. 411-429.
SÁNCHEZ, G., PRO, A. y VALCÁRCEL, M.V. (1997). La utilización de un modelo de planificación de unidades didácticas: el estudio de las disoluciones en la educación secundaria. Enseñanza de las Ciencias, 15(1), pp. 35-50.

SILVA, A.A. (1999). Didáctica da Física - perspectivas centradas na natureza da evolução conceptual. Porto: Edições ASA.

VERGNAUD, G. (1987). Les fonctions de l'action et de la symbolisation dans la formation des connaissances chez l'enfant, en Piaget, J., Mounoud, P. y Bronkart, J.P. (eds.). Encyclopédie de la Pléiade Psycologie, pp. 821-844. París: Gallimard.

VERGNAUD, G. (1991). La théorie des champs conceptuels. Recherches en Didactique des Mathématiques, 10(23), pp. 133-170.

VIENNOT, L. (1996). Raisonner en physique. La part du sens commun. París-Bruselas: De Boeck Université.

VYGOTSKY, L.S. (1934). Pensamento e linguagem. Sao Paulo: Martins Fontes Editora.

WEIL-BARAIS, A. (1995). La formation des connaissances en sciences expérimentales, en Gaonac'h, D. y Golder, C. (eds.). Manuel de Psychologie pour l'Enseignement, pp. 410-437. París: Hachette Éducation.

WELLINGTON, J. (1994). Secondary Science. Contemporary Issues and Practical Approaches. Londres: Routledge.

[Artículo recibido en julio de 2000 y aceptado en marzo de 2001.] 


\section{ANEXO A}

TE núm. 1. Medición de magnitudes físicas (Lopes et al., 2000)

\section{INTRODUCCIÓN}

La medición es fundamental en toda ciencia. Medición es, como el propio nombre indica, el acto de medir. Se trata de un conjunto de operaciones destinadas a determinar el valor de la magnitud, también denominado medida de la magnitud. La medida es, por tanto, el resultado de una medición.

Si las mediciones no fuesen efectuadas cuidadosamente, entonces los resultados no podrían merecer gran confianza. Como ninguna medida, incluso efectuada con cuidado, está exenta de error, es importante conocer el error (la incerteza) que afecta a una medida para saber hasta qué punto se puede confiar en el resultado obtenido.

\section{TAREA}

Con relación a todo ello vamos a realizar la siguiente tarea: estudiar aparatos de medida, medir y registrar magnitudes, estimar errores cometidos en las mediciones.

\section{Información}

Los errores cometidos tienen dos naturalezas:

Errores sistemáticos. Son errores que se cometen siempre del mismo modo y afectan a los resultados siempre en idéntico sentido. Estos errores pueden ser debidos a deficiencias del método, del aparato, del observador; de las condiciones en las que la medición es efectuada.

Errores fortuitos o accidentales. Son errores que suceden ocasionalmente en una medición y no ocurren siempre del mismo modo. Pueden resultar de la posición inadecuada del objeto a medir o de errores de paralaje cometidos en las lecturas. Como estos errores afectan al resultado, en un sentido o en otro, pueden minimizarse si se trabajan cuidadosamente efectuando varias mediciones de modo que se pueda calcular el valor más probable de la magnitud medida: $\overline{\mathrm{x}}=\frac{\mathrm{x}_{1}+\mathrm{x}_{2}+\ldots+\mathrm{x}_{\mathrm{n}}}{\mathrm{n}}$. Cuanto mayor sea el número de mediciones, menor será el error.

Las mediciones sólo pueden ser realizadas si existen instrumentos y unidades.

\section{MEDICIÓN DE LONGITUD}

\section{Material necesario}

Mesa, cinta métrica.

\section{Modo de proceder}

Estudio de la cinta métrica:

Observa la escala en la que está graduada la cinta métrica e indica los límites entre los que puede ser utilizada (alcance).

Indica el valor de la menor división de la escala (sensibilidad del instrumento).

Cuestión de reflexión: ¿Conseguirá medir, con esta cinta métrica, una longitud de 5,37 cm?

Indica la fracción de la menor división de la escala que puede ser leída por estimativa.

Indica el valor de la incerteza o error en la lectura de la escala (suele considerarse la mitad de la menor división de la escala -incerteza o error de lectura).

\section{Medición de la longitud de la tabla de su mesa de trabajo}

Información 1: Cuando se procede a la lectura de un valor numérico en un instrumento de medida, éste se expresará a través de números significativos (los exactos y uno más aproximado [leído por estimativa] y pasará a tener significado físico si tiene unidades.

Con la cinta métrica mide la longitud de tu mesa de trabajo y registra $\left(l_{1}=\right.$

Realiza la operación anterior dos veces más $\left(l_{2}=\right.$. $; l_{3}=. . . .$. ....).

Calcula el valor más probable de la longitud (media aritmética de los varios valores obtenidos) $\bar{l}=\frac{l_{1}+l_{2}+\ldots+l_{3}}{3}$ 
Determina los desvíos en relación con el valor medio de cada una de las lecturas efectuadas $\delta_{1}=l_{1}-\bar{l} ; \delta_{2}=l_{2}-\bar{l} ; \delta_{3}=l_{3}-\bar{l}$

Organiza estos resultados en un cuadro.

\begin{tabular}{|c|c|c|c|c|}
\hline & Longitud /cm & Desvío /cm & Módulo del desvío /cm & $\begin{array}{l}\text { Incerteza o error } \\
\text { de observación /cm }\end{array}$ \\
\hline & $\begin{array}{l}l_{1}= \\
l_{2}= \\
l_{3}=\end{array}$ & $\delta_{1}=$ & $\left|\delta_{1}\right|=$ & \\
\hline $\begin{array}{l}\text { Valor más } \\
\text { probable }\end{array}$ & $\bar{l}=$ & & & \\
\hline
\end{tabular}

Información 2: El módulo del mayor desvío es la incerteza o error de la medida de la longitud (incerteza o error de observación) Medida de la longitud (resultado de la medición)

Información 3: La medida de la longitud = valor más probable \pm incerteza o error (unidad). Se considera para incerteza o error, $\Delta \mathrm{l}$, de la medida la mayor de las incertezas (de observación y lectura) $l=\bar{l} \pm \Delta l$ (unidad).

El verdadero valor de la longitud está, así, comprendido entre $\bar{l}-\Delta l$ (unidad) y $\bar{l}+\Delta l$ (unidad).

Indica la medida de la longitud

Cuestiones de reflexión:

¿Cómo representarías la medida de esta longitud dentro de una escala?

¿Podrías medir con este instrumento una longitud de $3 \mathrm{~m}$ ?

\section{MEDICIÓN DE FUERZAS}

Material necesario

Tabla rectangular $(50 \mathrm{~cm}$ de longitud) y un tornillo en una extremidad, un muelle en hélice, un dinamómetro y una regla.

\section{Modo de proceder}

Estudio del dinamómetro:

Indica el alcance y la sensibilidad del dinamómetro.

Indica el error de lectura asociado a este instrumento.

\section{Cuestión de reflexión:}

¿Conseguirá medir, con este dinamómetro, una fuerza de intensidad 2,40 N?

Medición de la fuerza ejercida sobre un muelle elástico, que provoca en éste una deformación.

Intercala el muelle entre el tornillo y el gancho del dinamómetro. Sobre la tabla aplica la regla que te permite medir el estiramiento sufrido por el muelle.

Sujeta por la extremidad del dinamómetro y lee en la regla el valor correspondiente a la posición inicial de la extremidad del muelle, $x_{0}$, (sin deformación, $\left.F=0\right)$.

Disloca el dinamómetro hasta provocar en el muelle (M) una pequeña deformación y lee el valor indicado en el dinamómetro, $F_{l}$, en la regla, $x_{1}$, y anota el resultado.

Repite durante diez veces la operación anterior, manteniendo $x_{l}$. 
Organiza un cuadro con las medidas obtenidas.

\begin{tabular}{|c|c|c|c|c|}
\hline $\mathrm{X}_{0} / \mathrm{cm}$ & $\mathrm{X}_{1} / \mathrm{cm}$ & $\begin{array}{c}\text { Deformación } \\
\Delta \mathrm{x}=\mathrm{x}_{1}-\mathrm{x}_{0} / \mathrm{cm}\end{array}$ & $\begin{array}{c}\text { Intensidad de la fuerza } \\
\text { deformadora } \mathrm{F} / \mathrm{N}\end{array}$ & $\begin{array}{c}\text { Incerteza o error } \\
\text { de observación } / \mathrm{N}\end{array}$ \\
\hline $\mathrm{X}_{0}=$ & $\mathrm{X}_{1}=$ & $\Delta \mathrm{x}_{1}=$ & $\mathrm{F}_{1}=$ & \\
$\mathrm{X}_{0}=$ & $\mathrm{X}_{1}=$ & $\Delta \mathrm{x}_{1}=$ & $\mathrm{F}_{2}=$ & \\
$\ldots$ & $\cdots$ & $\cdots$ & $\cdots$ & \\
\hline
\end{tabular}

Indica la medida de la fuerza.

Cuestiones de reflexión

¿Por qué, en este caso, el error de observación es superior al error de lectura?

¿Cómo representarías la medida de esta fuerza en una escala?

¿Podrías medir con este dinamómetro una fuerza de intensidad 1,54 N?

\section{Comunicación de los resultados}

Haz una síntesis de lo que has aprendido con relación a la medición de magnitudes físicas y señala lo que es importante en relación con este asunto. Esta síntesis será un modo eficaz de conservar una memoria sobre un esfuerzo que te ha permitido comprender mejor la medición de magnitudes físicas. 


\section{ANEXO B}

Auxiliar didáctico del desarrollo conceptual a través del trabajo experimental ADDCTE del TE núm. 1 (Lopes et al., 2000)

1) Situación física y particularidades:

Medición directa de magnitudes físicas en situaciones corrientes y en situaciones de laboratorio.

Particularidades: Las mediciones son efectuadas con ayuda de aparatos que tienen limitaciones propias.

2) Conceptos en juego:

\begin{tabular}{|l|l|}
\hline Conceptos & Aspectos operacionales de los conceptos \\
\hline Medición & \\
Medida & Cálculo del valor más probable \\
Magnitud física & Cálculo del error \\
Error (sistemático y fortuito) & Representación de una medida (a nivel gráfico y a nivel numérico) \\
Valor más probable & \\
Desvío & \\
\hline
\end{tabular}

3) Modelo implícito:

La medición de una magnitud física es una operación que nunca nos conduce al valor exacto de una medida, pero podemos saber cuándo estamos cerca de ese valor si eliminamos todo tipo de errores sistemáticos.

4) Desarrollo conceptual durante la implantación del protocolo:

\begin{tabular}{|c|c|c|}
\hline Estructura del protocolo & Sugestiones de exploración & Desarrollo conceptual \\
\hline Problema & $\begin{array}{l}\text { Por qué medir magnitudes físicas } \\
\text { Discusión de los diferentes } \\
\text { tipos de errores }\end{array}$ & $\begin{array}{l}\text { Modelo implícito } \\
\text { Proceso de cuantificación } \\
\text { de propiedades físicas }\end{array}$ \\
\hline $\begin{array}{l}\text { Estudio de los aparatos } \\
\text { de medida }\end{array}$ & $\begin{array}{l}\text { Cuestiones de reflexión } \\
\text { Verificar si los alumnos están indicando } \\
\text { correctamente la precisión } \\
\text { y alcance de los aparatos }\end{array}$ & $\begin{array}{l}\text { Proceso de medición: } \\
\text { - repetición de ciertas operaciones } \\
\text { - magnitud física } \\
\text { - error } \\
\text { - cálculo del valor más probable } \\
\text { - modelo implícito }\end{array}$ \\
\hline Medición & $\begin{array}{l}\text { Cuestiones de reflexión } \\
\text { Verificar si hacen correctamente el cálculo } \\
\text { del valor más probable, } \\
\text { del error y la representación } \\
\text { de la medida de la respectiva magnitud }\end{array}$ & $\begin{array}{l}\text { Proceso de estimar errores: } \\
\text { - error } \\
\text { - valor más probable } \\
\text { - cálculo del error } \\
\text { Procesos de representar } \\
\text { la medida de la magnitud } \\
\text { (analítico y gráfico) }\end{array}$ \\
\hline
\end{tabular}

